\title{
Efficacy of a Sleep Quality Intervention in People With Low Back Pain: Protocol for a Feasibility Randomized Co-Twin Controlled Trial
}

\author{
Marina B. Pinheiro, ${ }^{1}$ Kevin K. Ho, ${ }^{1}$ Manuela L. Ferreira, ${ }^{2}$ Kathryn M. Refshauge, ${ }^{1}$ Ron Grunstein, ${ }^{3}$ \\ John L. Hopper, ${ }^{4}$ Christopher G. Maher, ${ }^{5}$ Bart W. Koes, ${ }^{6}$ Juan R. Ordoñana, ${ }^{7}$ and Paulo H. Ferreira ${ }^{1}$ \\ ${ }^{1}$ Faculty of Health Sciences, The University of Sydney, Sydney, New South Wales, Australia \\ ${ }^{2}$ The George Institute for Global Health and Institute of Bone and Joint Research, The Kolling Institute, Sydney Medical \\ School, The University of Sydney, Sydney, New South Wales, Australia \\ ${ }^{3}$ CIRUS, Centre for Sleep and Chronobiology, Woolcock Institute of Medical Research, University of Sydney and Royal \\ Prince Alfred Hospital, NSW, Australia \\ ${ }^{4}$ Centre for Epidemiology and Biostatistics, Melbourne School of Population and Global Health, The University of \\ Melbourne, Melbourne, Victoria, Australia \\ ${ }^{5}$ The George Institute for Global Health, Sydney Medical School, The University of Sydney, Sydney, New South Wales, \\ Australia \\ ${ }^{6}$ Department of General Practice, Erasmus MC, Rotterdam, The Netherlands \\ ${ }^{7}$ Murcia Twin Registry, Department of Human Anatomy and Psychobiology, University of Murcia, and IMIB-Arrixaca, \\ Murcia, Spain
}

Poor sleep quality is highly prevalent in patients with low back pain (LBP) and is associated with high levels of pain, psychological distress, and physical disability. Studies have reported a bidirectional relationship between sleep problems and intensity of LBP. Accordingly, effective management of LBP should address sleep quality. In addition, genetics has been found to significantly affect the prevalence of both LBP and insomnia. Our study aims to establish the feasibility of a trial exploring the efficacy of a web-based sleep quality intervention in people with LBP, with the genetic influences being controlled for. 30 twins (15 complete pairs) with subacute or chronic LBP (>6 weeks) will be recruited from the Australian Twin Registry. Participants will be randomly assigned to one of the two groups with each twin within a pair receiving either an interactive web-based sleep intervention based on cognitive behavioral therapy principles (intervention) or a web-based education program (control) for 6 weeks. The feasibility of the trial will be investigated with regard to recruitment rate, feasibility of data collection and outcome measure completion, contamination of intervention, acceptability and experience of intervention, and sample size requirement for the full trial. Patient outcomes will be collected electronically at baseline, immediately post-treatment, and at 3-months' follow-up post-randomization. This trial employs a robust design that will effectively control for the influence of genetics on treatment effect. Additionally, this study addresses sleep quality, a significant but under-explored issue in LBP. Results will inform the design and implementation of the definitive trial.

Keywords: low back pain, sleep, insomnia, twins, cognitive behavioral therapy, internet, online, feasibility

Low back pain (LBP) is a highly prevalent and costly condition. In Australia, it is estimated that approximately 4 million people suffer from LBP at any one time (Australian Institute of Health and Welfare, 2004) and the estimates of prevalence around the world range from $22 \%$ to $65 \%$, depending on how the condition is defined (Walker, 2000). The treatment costs associated with LBP in Australia (a country of 24 million people) are close to $\$ 5$ billion per annum (Arthritis and Osteoporosis Victoria, 2013). LBP is commonly associated with other important health conditions, and the coexistence of other chronic conditions alongside LBP substantially increases the complexity of the management and care for these patients (Baumeister et al., 2012).

RECEIVEd 26 May 2016; ACCEPTEd 7 June 2016

ADDRESS FOR CORRESPONDENCE: Marina B. Pinheiro, Faculty of Health Sciences, The University of Sydney, 75 East Street, Lidcombe, Sydney NSW 2141, Australia. E-mail: mdeb9852@uni.sydney.edu.au 
Symptoms of insomnia are highly prevalent in patients with LBP. Approximately, 59\% of patients with LBP also have significant problems sleeping (Alsaadi et al., 2012), and the coexistence of these conditions is associated with higher levels of pain (Alsaadi et al., 2012), psychological distress, physical disability, fatigue, and daytime sleepiness (McCracken \& Iverson, 2002). Previous studies have suggested a bidirectional relationship between sleep problems and LBP intensity (Alsaadi et al., 2014a; Kelly et al., 2011). Insomnia is an important predictor of worse prognosis in terms of disability - patients with insomnia and back pain are at higher risk of going onto the disability pension than those without insomnia (Ropponen et al., 2013). Insomnia is also believed to lower pain thresholds and reduce people's mental capacity to deal with pain (Kundermann et al., 2004; Novak et al., 2004). On the other hand, the experience of pain also affects sleep quality. A previous study found that higher pain intensity during the day was associated with a decrease in the subsequent night's sleep quality (Alsaadi et al., 2014b). Additionally, it is likely that poor sleep quality adversely impacts the management of LBP: the most commonly prescribed treatment by clinicians for patients with LBP - exercise therapy (Freburger et al., 2009) — is likely to be affected by the consequences of insomnia, such as fatigue and daytime sleepiness, potentially leading to worse outcomes. Accordingly, it is plausible that effective management of LBP should address symptoms of insomnia.

The current treatment options for LBP offer only moderate effects at best (Machado et al., 2009). Previous randomized clinical trials have mainly focused on traditional intervention programs targeting specific spinal impairments, such as specific forms of exercise, and have shown limited effects (Ferreira et al., 2006; 2007). Treatment models focusing on associated comorbidities, such as insomnia, rather than the sole disease or disease-related impairment, have the potential to improve LBP outcomes and in the case of insomnia have not been previously investigated.

Behavioral interventions, such as cognitive behavioral therapy (CBT), are commonly prescribed for insomnia and have been shown to be effective in improving sleep quality (Riemann \& Perlis, 2009; Trauer et al., 2015). Previous studies have investigated the efficacy of CBT for sleep quality for patients with comorbid insomnia and osteoarthritis (Vitiello et al., 2009; 2014) or chronic pain (Jungquist et al., 2010; Tang et al., 2015), and found significant improvements in sleep outcomes as well as in pain and function. However, in these studies patients had to attend faceto-face treatment sessions, a treatment model that incurs high costs and limits the population's accessibility to the intervention. The use of an internet-based CBT program has been found to be effective for improving sleep quality (Espie et al., 2012; Seyffert et al., 2016) and overcomes the limitations of traditional face-to-face CBT interventions. The employment of an internet-based CBT program for patients suffering from LBP and poor sleep quality has not been pre- viously investigated and has great potential, considering the close relationship between insomnia and LBP.

Although randomized controlled trials are the gold standard design for investigating the effectiveness of an intervention, they do not explicitly take into consideration genetic and familial confounding. Genetic factors have a substantial influence in LBP, with heritability accounting for up to $67 \%$ of the variance in liability to LBP as a symptom and with the genetic component being higher for more recurrent and disabling LBP than acute and less disabling LBP (Ferreira et al., 2013). Similarly, genetic factors have been found to be associated with insomnia (Gottlieb et al., 2007), accounting for at least 33\% of the variance of sleep quality, sleep disturbance, and sleep pattern (Barclay et al., 2010; Heath et al., 1990; Watson et al., 2014). The randomized co-twin controlled trial design builds on the strengths of traditional trials design as it offers optimal matching to control for confounding, since genetics and a range of environmental variables that might contribute to the response to treatment are accounted for. This design has been successfully used in other areas such as the common cold (Martin et al., 1982), and osteoporosis (Hunter et al., 2000; Ronkainen et al., 2009), but to the best of our knowledge has never been used in LBP.

A randomized co-twin controlled trial investigating the efficacy of a web-based CBT treatment could reveal promising results that could be implemented on a population level. As the proposed study design (a randomized co-twin controlled trial) and intervention (web-based CBT program) are clearly innovative, the aim of this study is to assess the feasibility of this study and determine the optimal approach to conduct a fully powered randomized controlled trial.

The specific aims of this study are to investigate: (1) the rate of recruitment of adult twins registered at the Australian Twin Registry (ATR) with LBP to participate in the trial, (2) the feasibility of data collection and outcome measure completion, (3) contamination of intervention among twins, (4) acceptability and experience of the intervention, and (5) the sample size required for the full trial.

\section{Materials and Methods \\ Study Design}

This is a feasibility study of a randomized co-twin controlled trial with concealed allocation and blinded treatment administrators and trial statistician (Everitt \& Howell, 2005). This study has been approved by the Research Ethics Committee of the University of Sydney (2015/386) and been registered at the Australian New Zealand Clinical Trials Registry (ACTRN12615000672550). The protocol has been written following the SPIRIT statement to enhance transparency of content and completeness (Chan et al., 2013). The findings of the trial will be reported following the CONSORT statement and the TIDieR checklist (Altman et al., 2001; Hoffmann et al., 2014). 


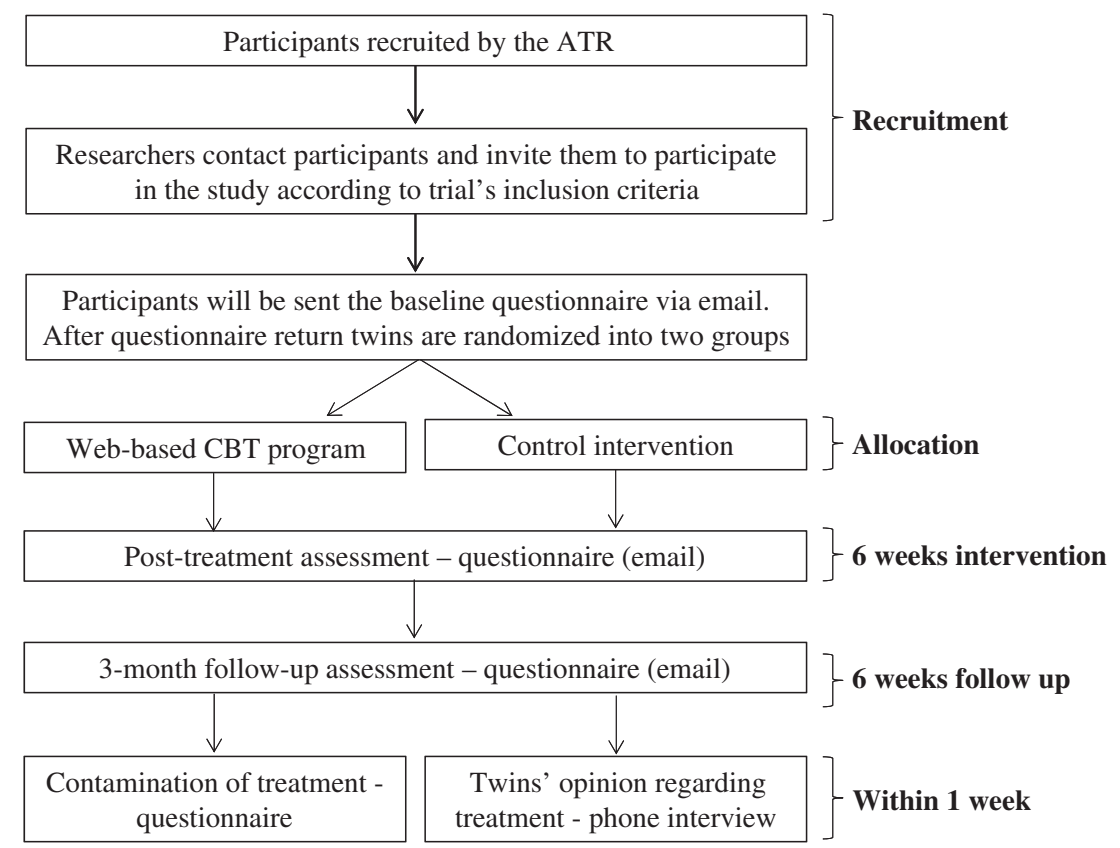

FIGURE 1

Study design, recruitment process, and flow of participants.

Note: ATR = Australian Twin Registry, CBT = cognitive behavioral therapy.

\section{Study Overview}

Monozygotic (MZ) and dizygotic (DZ) twins with subacute or chronic LBP will be enrolled. Participants will be randomly assigned to one of two groups and each twin within a pair will receive either a web-based CBT sleep intervention or a control intervention, with randomization taking place within the pair. The intervention will be an interactive web-based sleep intervention based on CBT principles (intervention group), whereas the control intervention will be a web-based education program (control group), with both interventions lasting for 6 weeks. Outcomes will be measured at baseline, immediately post-treatment, and at a 3-month follow-up post-randomization. Participants will be required to answer a questionnaire 1 week after the end of the study containing questions about contamination of intervention. Additionally, twins will answer a telephone interview to investigate their opinion regarding the webbased sleep intervention. All assessment, treatment, and data-collection procedures will be conducted online and all enquiries will be managed electronically or via telephone (Figure 1).

\section{Participants}

A total of 30 twins (15 pairs) with current e-mail address in the ATR database and concordant for LBP will be recruited. Twins will be contacted through an e-mail approach coordinated by the ATR. To be included in the study both twins within a pair must meet all of the following criteria:

1. aged between 18 and 65 years
2. present with LBP of at least 6 weeks duration and not currently seeking care for their LBP

3. at least a score of 3 on a $0-10$ numerical pain scale (Scrimshaw \& Maher, 2001)

4. presenting with symptoms of insomnia, based on the DSM-5 criteria for persistent Insomnia disorder (Reynolds et al., 2010) assessed by Sleep Condition Indicator (SCI). The SCI is valid, reliable and sensitive to change. The items assess four dimensions: sleep continuity (getting to sleep, remaining asleep); sleep satisfaction (sleep quality, troubled by sleep in general); symptom severity (nights per week, duration of problem); and daytime consequences (personal functioning, daytime performance). The SCI generates scores in the range $0-32$, with lower scores indicating worse sleep. A score of $\leq 16$ is indicative of clinically significant insomnia (Espie et al., 2014). Twins with a score $<24$ will be included in our study

5. have current access to the internet with a device with a speaker, such as a computer or tablet.

Participants will be excluded, if they

1. have a known or suspected serious spinal pathology (fracture, metastatic, inflammatory or infective diseases, and widespread neurological disorder)

2. had spinal surgery in the past 12 months

3. are currently using prescribed medication or receiving any other prescribed treatment for insomnia or for depression

4. are pregnant or lactating women 


\section{TABLE 1}

Summary of Interventions

\begin{tabular}{|c|c|c|}
\hline & Web-based cognitive behavioral therapy (Sleepio) & Control group intervention \\
\hline Intervention content & $\begin{array}{l}\text { - Sleep information } \\
\text { - Behavioral techniques (e.g., sleep restriction, stimulus control) } \\
\text { - Cognitive techniques (e.g., restructuring, imagery, articulatory } \\
\text { suppression, paradoxical intention, and mindfulness } \\
\text { strategies) } \\
\text { - Sleep hygiene } \\
\text { - Relaxation strategies } \\
\text { - Underlying algorithms feed the delivery of information, } \\
\text { support, and advice in a personally tailored manner }\end{array}$ & $\begin{array}{l}\text { - Sleep information (derived from the Sleepio library) } \\
\text { - Delivered by e-mail not in a personally tailored manner }\end{array}$ \\
\hline Duration & - 6 weeks (one online session/week) & - 6 weeks (one e-mail/week) \\
\hline Additional features & $\begin{array}{l}\text { - Delivered by an animated personal therapist (avatar) } \\
\text { - Personalized case file } \\
\text { - Automatic periodic sleep data calculation } \\
\text { - Goal setting and review } \\
\text { - } 24 / 7 \text { online access } \\
\text { - Automated web and e-mail support } \\
\text { - Access to video library and back catalogue of session content } \\
\text { and Wikipedia style articles } \\
\text { - Able to participate in a moderated social network/community } \\
\text { of users }\end{array}$ & $\begin{array}{l}\text { - N/A } \\
\text { - Participants allocated to this group will be offered the } \\
\text { web based CBT package at the end of the study. }\end{array}$ \\
\hline
\end{tabular}

Note: $\mathrm{N} / \mathrm{A}=$ not applicable.

5. present with severe or extremely severe symptoms of depression (score $>10$ ), anxiety (score $>7$ ), and stress (score $>12$ ) assessed by the Depression Anxiety Stress Scales (DASS-21; Henry \& Crawford, 2005)

6. are in 'poor' or 'very poor' physical or mental health (self-report)

7. have a diagnosis of substance use disorder (self-report assessed with a question in the screening questionnaire)

8. are shift workers

9. have not had their zygosity ascertained.

\section{Recruitment}

The ATR will send an invitation e-mail with information about the study to twins registered in the database. At the end of the information sheet, twins will be invited to answer a screening questionnaire. Complete twin pairs meeting all preliminary inclusion criteria will be invited to participate in the study and will receive the participant information statement and informed consent. Only those participants meeting the inclusion criteria and returning the informed consent will be included in the study. The trial coordinator will send the link to the web-based baseline questionnaire for those twins who met the inclusion criteria before randomization.

\section{Randomization}

Once both twins in a pair meet these inclusion criteria and agree to participate in the study, they will be randomized into one of two groups: one twin from each pair will be randomly allocated to each of the intervention groups, with randomization taking place within the pair. Allocation will be performed using a computer-generated random allocation schedule operated by a remote researcher and concealed from the main assessor of the study. Both twins within a pair will start the intervention at a synchronized time. Twins enrolled in the trial will be contacted by phone to be advised about their intervention allocation and to receive details about the intervention.

\section{Blinding}

Treatment administrators and the trial statistician will be blinded to group allocation. Blinding of participants to the active, experimental intervention will also be attempted. In order to check whether participants are blinded to allocation, at the end of the intervention period, participants will be asked 'Which intervention did you receive?', and they will be given two options: real (experimental) intervention or sham (control) intervention (Borkovec \& Nau, 1972; Smeets et al., 2008). Participants will be asked to rate the credibility of the intervention (measured with the Intervention Credibility Scale) after the first week of intervention.

\section{Sample Size}

As the aim of this study is to investigate the feasibility of a future randomized co-twin trial, no sample size estimation was performed (Browne, 1995; Thabane et al., 2010). Based on feasibility, we have established our sample size as 30 participants (15 complete twin pairs).

\section{Interventions}

Web-based CBT. Participants allocated to this group will receive a web-based CBT course comprised of six online sessions, once per week, with sessions unlocked weekly. The program is highly interactive and the content is delivered by an animated personal therapist (avatar), with automated web and e-mail support developed by Sleepio limited (see https://www.sleepio.com) (Table 1). Previous studies, including a randomized-controlled placebo trial, 
have shown the effectiveness of this program in improving sleep and associated daytime functioning of adults with insomnia (Espie et al., 2012). In this web-based program, underlying algorithms feed the delivery of information, support, and advice in a personally tailored manner. Each session has a minimum duration of $20 \mathrm{~min}$. Participants make a time for the session and are prompted via e-mail if they fail to attend the session. Participants are required to complete an electronic daily sleep diary over the intervention period. Each participant has an online case file, comprised of four sections: a progress review, a reminder of strategies to try out between sessions, an agreed sleep schedule, and a list of additional reading. It also includes additional relaxation strategies and advice on lifestyle and bedroom factors (sleep hygiene). Participants will have access to a video library and back catalogue of session content and Wikipedia style articles. Web-based CBT users will also be able to participate in a moderated social network/community of users. The CBT content is consistent with the literature, covering behavioral (e.g., sleep restriction, and stimulus control) and cognitive (e.g., thought restructuring, imagery, articulatory suppression, paradoxical intention, and mindfulness) strategies.

Control intervention. Participants in the control group will be given a web-based education program delivered by e-mail (Table 1). Participants will receive a weekly e-mail in newsletter format over a 6-week period (to allow for matching with the web-based CBT group), containing material with information regarding sleep, mainly extracted from the Sleepio library. The content of each weekly newsletter will be different, but all participants will receive the same material during the intervention period. This intervention was chosen for the control group because there is evidence that sleep hygiene alone is not effective in the treatment of chronic insomnia (Schutte-Rodin et al., 2008). Participants randomized to this group will be offered the web-based CBT package free of charge upon completion of the study, in case we find results favouring the intervention group.

\section{Feasibility Outcomes}

The main outcomes of this study include aspects related to the feasibility of a randomized co-twin controlled trial investigating a CBT sleep intervention in the treatment of patients with LBP. Records will be kept throughout the study and, in addition, a telephone interview will be used to investigate twins' opinions regarding the intervention and general aspects of the study. The interview will be conducted with twins who have concluded the study and we will attempt to also interview those who have dropped out. The phone interview will be recorded. Additionally, data on the relationship between the twins, including twin bond, communication, and time living together will also be collected using a questionnaire developed by the ATR. A detailed description of the assessment of each of the feasibility aspects is provided below.
Recruitment rate. Recruitment rate will be recorded during the entire recruitment process. Records will be kept regarding the number of twins screened for entry to the trial. When a twin is not admitted to the trial, the reason why he/she was ineligible for inclusion will be recorded. Similarly, if eligible, the reasons for declining participation in the trial will be noted. Consent rates will also be recorded.

Feasibility of data collection and outcome measure completion. The number of missing items for each questionnaire will be recorded. The number of participants completing the intervention program and answering the followup questionnaires will be recorded. The number of participants lost in each phase of the study will be noted and the reasons for dropping out will be recorded when possible. During the phone interview, participants will be asked about their opinion regarding the data collection method (electronic), understanding of study questionnaire and data collection tools, and length of time to complete the questionnaires.

Contamination of intervention. Despite all the advantages in conducting a randomized co-twin trial, contamination of intervention between twins in a pair could be a threat to the methodological quality of the trial. On completion of the study intervention, contamination of intervention will be discussed with participants during the telephone interview, and participants will be asked questions such as how difficult it was not to talk to the co-twin about the intervention (Table 2). To ensure interventions are delivered according to protocol, at the first treatment session twins will be invited to commit (or not) to the course. Twins will be asked not to discuss with their co-twins about the intervention they are receiving.

Acceptability and experience of intervention. According to the trial protocol, each participant in the experimental and control groups will be required to participate in a webbased program for a period of 6 weeks. All web-based interactions from the participants in the web-based CBT course will be electronically stored and participation activity will be assessed, using the following data: diary entries, session activities, engagement with the community, and adherence to tasks. Twins' opinions regarding the intervention, including the relevance of the intervention for them, will be investigated during the telephone interview.

Sample size requirement. To the best of our knowledge, this will be the first randomized co-twin controlled trial in LBP and also the first study to investigate an online CBT sleep quality intervention for LBP sufferers. Therefore, the results from this feasibility study will be used to generate data for sample size calculations for the definitive trial. The sample used for this feasibility study is representative of the target study population (twins registered with the ATR). 


\section{TABLE 2}

\section{Contamination of Treatment Questions}

1. Have you talked to your twin about the intervention you have received?

2. Please indicate how confident you are that your twin did not know about the intervention you were receiving on a scale of $0-10$, where 0 means not at all and 10 means very confident.

3. Were you aware of the intervention your twin was receiving?

4. Did you change your behavior/attitudes as a consequence of knowing about your twin's intervention?

Additionally, if this study proves to be feasible, the same inclusion and exclusion criteria will be used in the definitive trial. The quantitative data generated by this study will be supplemented by qualitative data.

\section{Patient Outcomes}

Patient outcomes will be measured at baseline, immediately post-intervention, and at a 3-month follow-up postrandomization. All data will be collected using electronic questionnaires. The patient outcomes will include the following:

1. pain self-efficacy, measured by the pain self-efficacy questionnaire (PSEQ; Nicholas et al., 1992)

2. function, assessed by the patient-specific functional scale (PSFS; Hall et al., 2011)

3. average pain intensity over the last week, assessed by a 0-10 pain scale (Scrimshaw \& Maher, 2001)

4. disability, assessed by the Roland Morris disability questionnaire (Roland \& Fairbank, 2000)

5. physical activity level, assessed by the International Physical Activity Questionnaire Short Form (IPAQ-SF; Lee et al., 2011)

6. insomnia severity, assessed by the insomnia severity index (Bastien et al., 2001)

7. subjective sleep efficiency: data obtained from the sleep diary, assessed at baseline, post-intervention and at 3 months post-randomization (Espie et al., 2012).

\section{Data Integrity}

Study data will be collected and managed using REDCap electronic data capture tools hosted at the University of Sydney (Harris et al., 2009). REDCap (Research Electronic Data Capture) is a secure, web-based application designed to support data capture for research studies. Data will be stored in spreadsheets and transferred to appropriate statistical software for analysis by an investigator blinded to group allocation. Spreadsheets will be regularly scrutinized for omissions and errors.

\section{Controlling Bias and Protocol Protection}

The design that will be employed in this study includes key methodological features that have been recognized as important to minimize bias in controlled trials: randomization, concealed allocation, specification of eligibility criteria, blinded treatment administrators, and trial statistician. The nature of the treatments precludes blinding of participants, although treatment credibility will be assessed us- ing the Credibility Scale. Participants will not be informed about which components of the treatment are active, and instead they will be informed that they will be randomized to two different web-based programs. Additionally, the co-twin design allows optimal matching to control for confounding, including genetic and environmental factors.

This trial has been registered, and to ensure that the study protocol is followed consistently, all personnel involved in the study will be appropriately trained. The nature of the web-based programs will ensure that all participants receive the same standardized intervention, as per protocol.

\section{Data Analysis}

Statistical analysis. Since this is a feasibility study, descriptive statistics will be used to explore the data. Analysis will focus on variability of the data (assessed by confidence intervals) rather than hypothesis testing since it is not powered appropriately to assess statistical significance. Treatment effects will be represented by summary (such as mean), and precision measures (such as confidence interval).

The co-twin design, with randomization taking place within the pair, offers high levels of adjustment and more precise estimates of effects than non-twin trials (Dupont, 1988; Visscher et al., 2008). Therefore, the sample size needed to identify the efficacy of an intervention is believed to be smaller than in a traditional non-twin trial. It is intended that the results of the study will enable determination of the appropriate sample size for a full trial (Thabane et al., 2010). We will explore, through descriptive analysis, trends in treatment effect sizes in twins reporting different levels of symptoms of insomnia. Additionally, we will attempt to investigate any trend in data variability in MZ twins compared to DZ Twins.

The data gathered from the telephone interview will be qualitatively analyzed. Main areas to be explored will be: reasons for participation in the study and for dropping out, relevance of the intervention for them, contamination of intervention, understanding of study questionnaire and data collection tools, twins' views on the data collection method, and barriers and motivators to participate in the study.

\section{Criteria for Feasibility}

Based on the results of this study one of the following decisions will be made: (1) study is not feasible, and therefore should not proceed to full trial; (2) study is feasible, but modifications are required; (3) study is feasible and 
no modifications are required (Thabane et al., 2010). The results of this feasibility study will be interpreted based on the following criteria to determine whether it is feasible to proceed to the full trial:

1. Recruitment rate of twins approached by the ATR: $10 \%$ recruitment rate considering all twins registered (the ATR currently has over 35,000 twin pairs registered), and at least $70 \%$ of all eligible pairs can be included. In case these values are not achieved, the reasons for declining participation will be analyzed and the recruitment approaches will be restructured. Additionally, the communication material used to approach twins will be checked and other methods of collecting data might be necessary, such as paper-based questionnaires, and use of incentives for participating in the study might be needed.

2. Feasibility of data collection and outcome measures completion: No more than $20 \%$ missing data for the outcome measures, and a minimum of $85 \%$ follow-up rate, based on the PEDro scale (www.pedro.org.au), will be used as a measure of feasibility of data collection and measure completion. If these targets are not achieved, the information collected during the interview regarding reasons for dropping out, twins' opinions regarding the method of data collection, understanding of study questionnaire and data collection tools, and amount of time required to answer the questionnaires will be used to modify the protocol.

3. Contamination of intervention: No more than $15 \%$ of twins being aware of the intervention their co-twins were receiving and indicating that they have changed their behavior as a consequence of this knowledge. If we find a contamination rate greater than $15 \%$, strategies to avoid it will be sought. For instance, we aim to explore whether contamination of intervention was associated with the level of bonding between twins, living together, or communication between twins. If these factors affect contamination they could be addressed and considered in the recruitment strategy of the full trial.

4. Acceptability and experience of intervention: Adherence rate of $75 \%$, based on the results of a previous study using Sleepio (Espie et al., 2012). The information collected during the phone interview regarding the twins' opinions in relation to the intervention will be used to support the interpretation of these results and to establish strategies to increase attendance rate.

5. Sample size requirement: Caution will be used when calculating the sample size required for the full trial, as results from feasibility studies can potentially mislead sample size calculation (Thabane et al., 2010). To avoid generating misleading results, we will supplement the information with qualitative data.
The criteria for the full study to be considered feasible or for requiring modification have been established above. The following criteria will be used for considering the study as not feasible: no apparent change in the outcomes with confidence intervals that include large negative values, or when the established criteria have not been met and no strategies can be used to overcome the issues (Thabane et al., 2010).

\section{Discussion}

There are many aspects of this trial that are innovative, such as investigation of an intervention approach that has not been tested in the target population (people with LBP) and the twin design. Therefore, the investigation of the feasibility of the study is imperative before planning the full trial, including such pioneering aspects.

This feasibility study includes specific and clear feasibility objectives, and clear analytic plans, and explicit criteria have been established to assess the feasibility of the full study. If this study is considered feasible in the current format, the same design and methods will be used for the full study. However, if modifications are required, they will be conducted according to the information collected in this feasibility study. This will ensure the quality and efficiency of the full trial. In the case that the study is considered as not feasible, the full study will not be conducted, saving resources that otherwise would be spent unnecessarily.

This study will represent a major advance in the field because symptoms of insomnia are strongly associated with chronic LBP and other musculoskeletal conditions, and the use of a co-twin design has the potential to provide precise estimates of treatment effects in LBP. Results will have a substantial impact in the identification of a new intervention that could be directly translated into the management of LBP.

This study will investigate the feasibility of a randomized co-twin trial investigating the efficacy of a web-based CBT program for insomnia to improve sleep and LBP outcomes in people with subacute or chronic LBP and symptoms of insomnia. Our findings will inform the preliminary efficacy of a sleep intervention for LBP, and the feasibility of conducting a definitive randomized co-twin trial.

\section{Acknowledgments}

Sleepio Limited for providing the web-based CBT intervention. The Australian Twins Registry for their assistance in the recruitment process. Trial registration: Australian New Zealand Clinical Trials Registry: ACTRN12615000672550. This research received no specific grant from any funding agency, commercial or not-for-profit sectors. MLF is supported by Sydney Medical Foundation/Sydney Medical School. 


\section{Conflicts of Interests}

None

\section{Details of Ethical Approval}

The authors assert that all procedures contributing to this work comply with the ethical standard of the relevant national and institutional committees on human experimentation and with the Helsinki Declaration of 1975, as revised in 2008.

\section{References}

Alsaadi, S. M., McAuley, J. H., Hush, J. M., Lo, S., Bartlett, D. J., Grunstein, R. R., \& Maher, C. G. (2014a). The bidirectional relationship between pain intensity and sleep disturbance/quality in patients with low back pain. Clinical Journal of Pain, 30, 755-765.

Alsaadi, S. M., McAuley, J. H., Hush, J. M., Lo, S., Lin, C. W. C., Williams, C. M., \& Maher, C. G. (2014b). Poor sleep quality is strongly associated with subsequent pain intensity in patients with acute low back pain. Arthritis \& Rheumatology, $66,1388-1394$.

Alsaadi, S. M., McAuley, J. H., Hush, J. M., \& Maher, C. G. (2012). Erratum to: Prevalence of sleep disturbance in patients with low back pain. European Spine Journal, 21, 554560 .

Altman, D. G., Schulz, K. F., Moher, D., Egger, M., Davidoff, F., Elbourne, D., ... Consort, G. (2001). The revised CONSORT statement for reporting randomized trials: Explanation and elaboration. Annals of Internal Medicine, 134, 663694.

Arthritis and Osteoporosis Victoria. (2013). A problem worth solving. The rising cost of musculoskeletal conditions in Australia. Elsternwick: Arthritis and Osteoporosis Victoria.

Australian Institute of Health and Welfare. (2004). Eighth biennial health report of the Australian institute of health and welfare. Canberra, Australia: Author.

Barclay, N. L., Eley, T. C., Buysse, D. J., Rijsdijk, F. V., \& Gregory, A. M. (2010). Genetic and environmental influences on different components of the Pittsburgh sleep quality index and their overlap. Sleep: Journal of Sleep and Sleep Disorders Research, 33, 659-668.

Bastien, C. H., Vallieres, A., \& Morin, C. M. (2001). Validation of the insomnia severity index as an outcome measure for insomnia research. Sleep Medicine, 2, 297-307.

Baumeister, H., Knecht, A., \& Hutter, N. (2012). Direct and indirect costs in persons with chronic back pain and comorbid mental disorders - A systematic review. Journal of Psychosomatic Research, 73, 79-85.

Borkovec, T. D., \& Nau, S. D. (1972). Credibility of analogue therapy rationales. Journal of Behavior Therapy and Experimental Psychiatry, 3, 257-260.

Browne, R. H. (1995). On the use of a pilot sample for sample size determination. Statistics in Medicine, 14, 1933-1940.

Chan, A. W., Tetzlaff, J. M., Altman, D. G., Laupacis, A., Gotzsche, P. C., Krleza-Jeric, K., ... Moher, D. (2013).
SPIRIT 2013 statement: Defining standard protocol items for clinical trials. Annals of Internal Medicine, 158, 200207.

Dupont, W. D. (1988). Power calculations for matched casecontrol studies. Biometrics, 44, 1157-1168.

Espie, C. A., Kyle, S. D., Hames, P., Gardani, M., Fleming, L., \& Cape, J. (2014). The sleep condition indicator: A clinical screening tool to evaluate insomnia disorder. BMJ Open, 4, e004183.

Espie, C. A., Kyle, S. D., Williams, C., Ong, J. C., Douglas, N. J., Hames, P., \& Brown, J.S. (2012). A randomized, placebo-controlled trial of online cognitive behavioral therapy for chronic insomnia disorder delivered via an automated media-rich web application. Sleep, 35, 769781.

Everitt, B. S., \& Howell, D. C. (2005). Co-twin control methods. In B. S. Everitt (Ed.), Encyclopedia of statistics in behavioral science. Vol. 1 (pp. 415-418). West Sussex, UK: Wiley.

Ferreira, M. L., Ferreira, P. H., Latimer, J., Herbert, R. D., Hodges, P. W., Jennings, M. D., ... Refshauge, K. M. (2007). Comparison of general exercise, motor control exercise and spinal manipulative therapy for chronic low back pain: A randomized trial. Pain, 131, 31-37.

Ferreira, P. H., Beckenkamp, P., Maher, C. G., Hopper, J. L., \& Ferreira, M. L. (2013). Nature or nurture in low back pain? Results of a systematic review of studies based on twin samples. European Journal of Pain, 17, 957-971.

Ferreira, P. H., Ferreira, M. L., Maher, C. G., Herbert, R. D., \& Refshauge, K. (2006). Specific stabilisation exercise for spinal and pelvic pain: A systematic review. Australian Journal of Physiotherapy, 52, 79-88.

Freburger, J. K., Carey, T. S., Holmes, G. M., Wallace, A. S., Castel, L. D., Darter, J. D., \& Jackman, A. M. (2009). Exercise prescription for chronic back or neck pain: Who prescribes it? Who gets it? What is prescribed?. Arthritis \& Rheumatology, 61, 192-200.

Gottlieb, D. J., O’Connor, G. T., \& Wilk, J. B. (2007). Genomewide association of sleep and circadian phenotypes. BMC Medical Genetics, 8, S9.

Hall, A. M., Maher, C. G., Latimer, J., Ferreira, M. L., \& Costa, L. O. (2011). The patient-specific functional scale is more responsive than the Roland Morris disability questionnaire when activity limitation is low. European Spine Journal, 20, 79-86.

Harris, P. A., Taylor, R., Thielke, R., Payne, J., Gonzalez, N., \& Conde, J. G. (2009). Research Electronic Data Capture (REDCap) - A metadata-driven methodology and workflow process for providing translational research informatics support. Journal of Biomedical Informatics, 42, 377381.

Heath, A. C., Kendler, K. S., Eaves, L. J., \& Martin, N. G. (1990). Evidence for genetic influences on sleep disturbance and sleep pattern in twins. Sleep, 13, 318-335.

Henry, J. D., \& Crawford, J. R. (2005). The short-form version of the Depression Anxiety Stress Scales (DASS-21): Construct validity and normative data in a large non-clinical sample. British Journal of Clinical Psychology, 44, 227239. 
Hoffmann, T. C., Glasziou, P. P., Boutron, I., Milne, R., Perera, R., Moher, D., ... Michie, S. (2014). Better reporting of interventions: Template for intervention description and replication (TIDieR) checklist and guide. British Medical Journal, 348, g1687.

Hunter, D., Major, P., Arden, N., Swaminathan, R., Andrew, T., MacGregor, A. J., ... Spector, T. D. (2000). A randomized controlled trial of vitamin D supplementation on preventing postmenopausal bone loss and modifying bone metabolism using identical twin pairs. Journal of Bone and Mineral Research, 15, 2276-2283.

Jungquist, C. R., O’Brien, C., Matteson-Rusby, S., Smith, M. T., Pigeon, W. R., Xia, Y., ... Perlis, M. L. (2010). The efficacy of cognitive-behavioral therapy for insomnia in patients with chronic pain. Sleep Medicine, 11, 302-309.

Kelly, G. A., Blake, C., Power, C. K., O’Keeffe, D., \& Fullen, B. M. (2011). The association between chronic low back pain and sleep: A systematic review. Clinical Journal of Pain, 27, 169-181.

Kundermann, B., Krieg, J. C., Schreiber, W., \& Lautenbacher, S. (2004). The effect of sleep deprivation on pain. Pain Research and Management, 9, 25-32.

Lee, P. H., Macfarlane, D. J., Lam, T. H., \& Stewart, S. M. (2011). Validity of the International Physical Activity Questionnaire Short Form (IPAQ-SF): A systematic review. International Journal of Behavioral Nutrition and Physical Activity, 8, 115.

Machado, L. A., Kamper, S. J., Herbert, R. D., Maher, C. G., \& McAuley, J. H. (2009). Analgesic effects of treatments for non-specific low back pain: A meta-analysis of placebocontrolled randomized trials. Rheumatology (Oxford), 48, 520-527.

Martin, N. G., Carr, A. B., Oakeshott, J. G., \& Clark, P. (1982). Co-twin control studies: Vitamin $\mathrm{C}$ and the common cold. Progress in Clinical and Biological Research, 103 Pt A, 365373.

McCracken, L. M., \& Iverson, G. L. (2002). Disrupted sleep patterns and daily functioning in patients with chronic pain. Pain Research and Management, 7, 75-79.

Nicholas, M. K., Wilson, P. H., \& Goyen, J. (1992). Comparison of cognitive-behavioral group treatment and an alternative non-psychological treatment for chronic low back pain. Pain, 48, 339-347.

Novak, M., Mucsi, I., Shapiro, C. M., Rethelyi, J., \& Kopp, M. S. (2004). Increased utilization of health services by insomniacs - An epidemiological perspective. Journal of Psychosomatic Research, 56, 527-536.

Reynolds, C. F., 3rd, Redline, S., Workgroup, D.-V. S.-W. D., \& Advisors. (2010). The DSM-5 sleep-wake disorders nosology: An update and an invitation to the sleep community. Journal of Clinical Sleep Medicine, 6, 9-10.

Riemann, D., \& Perlis, M. L. (2009). The treatments of chronic insomnia: A review of benzodiazepine receptor agonists and psychological and behavioral therapies. Sleep Medicine Reviews, 13, 205-214.

Roland, M., \& Fairbank, J. (2000). The Roland-Morris disability questionnaire and the oswestry disability questionnaire. Spine (Phila Pa 1976), 25, 3115-3124.
Ronkainen, P. H., Kovanen, V., Alen, M., Pollanen, E., Palonen, E. M., Ankarberg-Lindgren, C., ... Sipila, S. (2009). Postmenopausal hormone replacement therapy modifies skeletal muscle composition and function: A study with monozygotic twin pairs. Journal of Applied Physiology (1985), 107, 25-33.

Ropponen, A., Silventoinen, K., Hublin, C., Svedberg, P., Koskenvuo, M., \& Kaprio, J. (2013). Sleep patterns as predictors for disability pension due to low back diagnoses: A 23-year longitudinal study of Finnish twins. Sleep, 36, 891897.

Schutte-Rodin, S., Broch, L., Buysse, D., Dorsey, C., \& Sateia, M. (2008). Clinical guideline for the evaluation and management of chronic insomnia in adults. Journal of Clinical Sleep Medicine, 4, 487-504.

Scrimshaw, S. V., \& Maher, C. (2001). Responsiveness of visual analogue and McGill pain scale measures. Journal of Manipulative and Physiological Therapeutics, 24, 501-504.

Seyffert, M., Lagisetty, P., Landgraf, J., Chopra, V., Pfeiffer, P. N., Conte, M. L., \& Rogers, M. A. M. (2016). Internetdelivered cognitive behavioral therapy to treat insomnia: A systematic review and meta-analysis. PLoS ONE, 11, e0149139.

Smeets, R. J., Beelen, S., Goossens, M. E., Schouten, E. G., Knottnerus, J. A., \& Vlaeyen, J. W. (2008). Treatment expectancy and credibility are associated with the outcome of both physical and cognitive-behavioral treatment in chronic low back pain. Clinical Journal of Pain, 24, 305-315.

Tang, N. K., Lereya, S. T., Boulton, H., Miller, M. A., Wolke, D., \& Cappuccio, F. P. (2015). Nonpharmacological treatments of insomnia for long-term painful conditions: A systematic review and meta-analysis of patient-reported outcomes in randomized controlled trials. Sleep, 38, 1751-1764.

Thabane, L., Ma, J., Chu, R., Cheng, J., Ismaila, A., Rios, L. P., ... Goldsmith, C. H. (2010). A tutorial on pilot studies: The what, why and how. BMC Medical Research Methodology, 10, 1 .

Trauer, J. M., Qian, M. Y., Doyle, J. S., Rajaratnam, S. M., \& Cunnington, D. (2015). Cognitive behavioral therapy for chronic insomnia: A systematic review and metaanalysiscognitive behavioral therapy for chronic insomnia. Annals of Internal Medicine, 163, 191-204.

Visscher, P. M., Gordon, S., \& Neale, M. C. (2008). Power of the classical twin design revisited: II detection of common environmental variance. Twin Research and Human Genetics, 11, 48-54.

Vitiello, M. V., McCurry, S. M., Shortreed, S. M., Baker, L. D., Rybarczyk, B. D., Keefe, F. J., \& Von Korff, M. (2014). Shortterm improvement in insomnia symptoms predicts longterm improvements in sleep, pain, and fatigue in older adults with comorbid osteoarthritis and insomnia. Pain, 155, 1547-1554.

Vitiello, M. V., Rybarczyk, B., Von Korff, M., \& Stepanski, E. J. (2009). Cognitive behavioral therapy for insomnia improves sleep and decreases pain in older adults with comorbid insomnia and osteoarthritis. Journal of Clinical Sleep Medicine, 5, 355-362. 
Walker, B.F. (2000). The prevalence of low back pain: A systematic review of the literature from 1966 to 1998. Journal of Spinal Disorders, 13, 205-217.
Watson, N. F., Harden, K. P., Buchwald, D., Vitiello, M. V., Pack, A. I., Strachan, E., \& Goldberg, J. (2014). Sleep duration and depressive symptoms: A gene-environment interaction. Sleep, 37, 351-358. 